\title{
Cost-Effective Optimization on a Two Demand Classes Inventory System
}

\author{
Raj Kumar Malligarjunan ${ }^{1}$
}

\begin{abstract}
This paper investigates impact of change of priority on a continuous review inventory system with a mixture of backorders and lost sales. The inter-arrival processes are assumed to be exponentially distributed. The objective is to minimize the anticipated total cost rate by simultaneously optimizing the maximum inventory level and backorders. An entropy-based uncertainty metric for evaluating the operating performance is demonstrated. The proposed modification offers the practical value of establishing how the long-run cost rate is reduced. Various system performance measures are derived.
\end{abstract}

Keywords Stochastic inventory $\cdot$ Poisson demands $\cdot$ Lost sales $\cdot$ Backorders $\cdot$ Multiple demand classes

Mathematics Subject Classification $90 \mathrm{~B} 05 \cdot 60 \mathrm{~J} 27$

\section{List of symbols}

$\begin{array}{ll}S & \text { Maximum inventory level } \\ s & \text { Maximum backorders } \\ \lambda_{1} & \text { Arrival rate of type-1(high priority) demands } \\ \lambda_{2} & \text { Arrival rate of type-2(low priority) demands } \\ L(t) & \text { On-hand inventory level at time } t \\ \phi_{i} & \text { Steady state probability at state } i \\ E(L) & \text { Mean of on-hand inventory } \\ E(B) & \text { Mean of backorders } \\ E(R) & \text { Expected reorder order } \\ E\left(R^{\prime}\right) & \text { Expected reorder order in priority scheme }\end{array}$

Raj Kumar Malligarjunan mrajmkumarm@gmail.com

1 Devanga Arts College, Aruppukottai, Tamilnadu, India 


$\begin{array}{ll}E(S) & \text { Expected shortage rate } \\ V(L) & \text { Variance of on-hand inventory } \\ V(B) & \text { Variance of backorders } \\ \Omega_{A} & \text { State space of inventory level } \\ \Omega_{B} & \text { State space of backorders } \\ m & \text { Expected time between two reorder times in priority case } \\ m^{*} & \text { Expected time between two reorder times in equal-priority case } \\ m^{L} & \text { Expected time between two reorder times in non-priority case } \\ F(t) & \text { Distribution of time of reorder process } \\ \alpha & \text { Initial probability vector } \\ h & \text { Holding cost per item per unit time } \\ K & \text { Setup cost per order } \\ c_{B} & \text { Backorder cost per customer per unit time } \\ c_{S} & \text { Losses in revenue per customer per unit time } \\ T C & \text { Total expected cost per unit time } \\ T C E & \text { Total expected cost per unit time in equal priority } \\ H(\Phi) & \text { Shannon entropy for } \Phi \\ \Delta T C & \text { Relative cost decrease }\end{array}$

\section{Introduction}

A matter that is regarded as more important than another is called as 'priority'. In many inventory settings, a supply firm wishes to provide different levels of service to different customers. For example, in a service-parts network, a customer can choose amongst different contracts, each with a different price and level of service. So, there is a need to study inventory systems with different demand types.

Arslan et al. [1] considered a single-product inventory system that serves multiple demand classes, which differ in their shortage costs or service-level requirements. They assumed a critical-level control policy, and a backorder clearing mechanism in which treat a backorder for a lower-priority class equivalent to a reserve-stock shortfall for the high-priority class. Tang et al. [2] considered a two-class model in which high priority was given to the backorder demand class, and low priority was given to the lost-sales demand class. The authors in [3] discuss a two-class system with a supply capacity, in which high priority is assigned to the class with lost-sales, whereas part of the unsatisfied demands are lost in the low priority class and the rest are backlogged for one period. They took for granted that the replenishment decision must first fully satisfy all previous backorders at the beginning of each period, with the remaining on-hand inventories used to satisfy new requirements.

Sana and Goyal [4] considered a stochastic economic order quantity (EOQ) model over a finite time horizon where uniform demand over the replenishment period is price dependent. The author developed the criterion for the optimal solution for the replenishment size such that the integrated expected profit is maximized. An intuitionistic fuzzy EOQ inventory model with backlogging was investigated using the score functions for the member and nonmembership functions by De and Sana [5]. Pal et al. [6] analyzed a single-period newsvendor model to determine the optimal order quantity where the customers balking occurs. The model allowed partial backlogging and permits part of the backlogged shortages to turn into lost sales. Sarkar and Saren [7] considered the strategy that supplier offers retailer a full trade-credit policy whereas retailer offers their customers a partial trade-credit policy. The 
main purpose was to minimize the retailers annual total cost with finite replenishment rate. A comparison between inventory followed by shortages model and shortages followed by inventory model with variable demand rate was studied by Khanra et al. [8]. The writing of Cárdenas-Barrón et. al. [9] provides a basis for new directions in inventory management research. For further related works, our readers may refer [10] and [11].

Isotupa [12] analysed a two demand classes inventory system with lost sales. She assumed that low priority customers are lost when the inventory level is low. Zhao and Lian [13] addressed a queueing-inventory system with two classes of customers in which the priority for service is decided by the server. Liu et al. [14] analysed a flexible service policies for an inventory system with two classes of customers. When the on-hand inventory drops to pre-determined safety level, arrival ordinary customers receive service at a fixed probability. Raj Kumar [15] considered a two demand classes perishable inventory system that consists of a single server. He analysed an entropy-based uncertainty metric for measuring the operating performance.

Lost sales are those selling opportunities that the shopkeeper has lost because an item was out of stock that caused him to lose the opportunity to sell. For example, product wanted not in the assortment, size out of stock, could not find product wanted, product out of stock, and did not receive help are some reasons for lost sales.

The primary cost control focus has been on enhancing the order processing system not only to eliminate excessive production of inventory but also to decrease the number of stock outs and lost sales. Lost sales are a difficult risk to put a monetary value on, but we all must understand the impact and risk. So, this work concentrates on lost sales. We would like to find what happens when there is no item in the stock and the impact of prioritization during stock out periods.

Moreover, it is well known that many systems operate subject to random occurrences. Entropy is a measure of randomness, flexibility, order/disorder and uncertainty. It appears in the laws that are fundamental to several fields of science for example data compression and transmission, ergodic and probability theory. For more details about entropy measures, see Cover and Thomas [16].

In this paper, we study a continuous review inventory system serving two demand classes, in which low priority is assigned to the class with lost sales, while high priority is assigned to the class with backorders. Compared with earlier works, our paper assumes, instead of loosing one type of customers, both types of demand classes are allowed during stock out period with equal priority. The results show that the modification is cost-effective.

The remaining part of this paper is prepared as follows: The Model is described in second section. The third section provides the analysis of the inventory level and the number of back orders at steady state. In fourth section, the analysis of reorder times is given. In fifth section, several related performance measures of significance are provided. Based on these measures, we calculate the long-run total expected cost function which is pseudo-convex in both parameters $s$ and $S$ and the results are compared with existing models. The measures on uncertainty is provided in sixth section. Some numerical results are given in the last Section.

\section{Model}

We consider a continuous review inventory system. The capacity to store inventory is $S$. The items are drawn one at a time as and when a demand occurs. There are two types of customers namely type- 1 and type- 2 . The customers arrive at the system independently according to 
two Poisson processes with rates $\lambda_{1}$ and $\lambda_{2}$. A shopkeeper is always available in the system who serves to the demands one by one.

If there is a shortage of $s$ units, an order for $S+s$ units is placed and the replenishment of stock is instantaneous.

When the inventory level is zero, the shopkeeper allows only one half of customers from the two demand classes and the other half are assumed to be lost.

\section{Steady-State Probability Distribution}

Suppose $L(t)$ denote the on-hand inventory level at time $t$,

$$
\phi_{i}(t)=P[L(t)=i]
$$

so that the steady state probability is

$$
\phi_{i}=\lim _{t \rightarrow \infty} \phi_{i}(t) .
$$

We have,

$$
\begin{aligned}
& \phi_{i}^{\prime}(t)=\lambda_{12}\left[\phi_{i+1}(t)-\phi_{i}(t)\right], \quad i=1,2, \ldots, S-1, \\
& \phi_{i}^{\prime}(t)=\lambda_{12}\left[\phi_{i+1}(t)-\frac{\phi_{i}(t)}{2}\right], \quad i=0, \\
& \phi_{i}^{\prime}(t)=\frac{\lambda_{12}}{2}\left[\phi_{i+1}(t)-\phi_{i}(t)\right], \quad i=-1,-2, \ldots,-s+1 .
\end{aligned}
$$

In the steady state

$$
\phi_{i}= \begin{cases}\phi_{0} & i=-1,-2, \ldots,-s+1, \\ \frac{\phi_{0}}{2} & i=1,2, \ldots, S .\end{cases}
$$

Now, multiplying $Z^{i}$ with $\phi_{i}$ and summing for all $i$, we get

$$
\begin{aligned}
\sum_{i=0}^{-s+1} \phi_{i} z^{i} & =\sum_{i=0}^{-s+1} \phi_{0} z^{i} \\
\text { and } \sum_{i=1}^{S} \phi_{i} z^{i} & =\sum_{i=1}^{S} \frac{\phi_{0}}{2} z^{i} .
\end{aligned}
$$

The probability generating function is then defined as

$$
\begin{aligned}
\Phi(z) & =\sum_{i=-s+1}^{S} \phi_{i} z^{i} \\
& =\phi_{0}\left[\sum_{i=1}^{S} \frac{z^{i}}{2}+\sum_{i=-s+1}^{0} z^{i}\right]
\end{aligned}
$$

and the value of $\phi_{0}$ can be obtained from $\lim _{z \rightarrow 1} \Phi(z)=1$ as

$$
\phi_{0}=\left[s+\frac{S}{2}\right]^{-1} \text {. }
$$


To obtain the mean and variance of on-hand inventory level, we define

$$
\Phi_{1}(z)=\sum_{i=1}^{S} \phi_{i} z^{i} .
$$

By taking derivatives of the above equation at $z=1$, we obtain the following:

$$
\text { and } \begin{aligned}
E(L) & =\phi_{0} \frac{S(S+1)}{4} \\
& =\sum_{i=2}^{S} i(i-1) \phi_{0} z^{i-2}-[E(L)]^{2} \\
& =\phi_{0} \frac{S(S+1)}{2}\left[\frac{4 S-1}{6}-\phi_{0} \frac{S(S+1)}{8}\right] .
\end{aligned}
$$

Similarly, to get the mean and variance of backorders, consider

$$
\Phi_{2}(z)=\sum_{i=1}^{s-1} \phi_{0} z^{-i} \text {. }
$$

Thus

$$
\begin{aligned}
E(B) & =\phi_{0} \frac{s(s-1)}{2} \\
\text { and } V(B) & =\phi_{0} \frac{s(s-1)}{6}\left[3 s-s^{2}+5\right] .
\end{aligned}
$$

We note that for $S=1$ and $s=1$, the process $\{L(t) ; t \geq 0\}$ is a continuous time Markov process with state space

$$
\begin{aligned}
\Omega & =\Omega_{A} \cup \Omega_{B} \\
& =\{-s+1,-s+2, \ldots, 0\} \cup\{1,2, \ldots, S\} .
\end{aligned}
$$

The Chapman-Kolmogorov forward equations are then given by

$$
\begin{array}{ll}
\phi_{11}^{\prime}(t)=-\lambda_{12}\left[\phi_{11}(t)+\frac{\phi_{10}(t)}{2}\right], & \phi_{11}(0)=1, \\
\phi_{10}^{\prime}(t)=\lambda_{12}\left[\phi_{11}(t)-\frac{\phi_{10}(t)}{2}\right], & \phi_{10}(0)=0, \\
\phi_{01}^{\prime}(t)=-\lambda_{12}\left[\phi_{01}(t)+\frac{\phi_{00}(t)}{2}\right], & \phi_{01}(0)=0, \\
\phi_{00}^{\prime}(t)=\lambda_{12}\left[\phi_{01}(t)-\frac{\phi_{00}(t)}{2}\right], & \phi_{00}(0)=1,
\end{array}
$$

We solve the first two forward equations as follows: We have

$$
\phi_{11}(t)+\phi_{10}(t)=\operatorname{Pr}(L(t)=0 \text { or } 1 / L(0)=1)=1 .
$$

Substituting for $\phi_{10}(t)$ in the first equation, we obtain

$$
\begin{aligned}
\phi_{11}^{\prime}(t) & =-\lambda_{12} \phi_{11}(t)+\left(1-\phi_{00}(t)\right) \frac{\lambda_{12}}{2} \\
& =\lambda_{12}\left(-\frac{3}{2} \phi_{11}(t)+\frac{1}{2}\right) .
\end{aligned}
$$


This is a non-homogeneous first order differential equation with constant coefficients. By solving we obtain

$$
\phi_{11}(t)=\frac{1}{3}\left(1+2 e^{-\frac{3}{2} \lambda_{12} t}\right) .
$$

Using the Eq. (3), we obtain

$$
\begin{aligned}
\phi_{10}(t) & =1-\phi_{11}(t) \\
& =\frac{2}{3}\left(1-e^{-\frac{3}{2} \lambda_{12} t}\right) .
\end{aligned}
$$

Similarly, we can solve the last two equations to obtain $\phi_{01}(t)$ and $\phi_{00}(t)$. Combining all these equations, the matrix $\Phi(t)$ is given by

$$
\Phi(t)=\left[\begin{array}{cc}
\frac{1}{3}\left(1+2 e^{-\frac{3}{2} \lambda_{12} t}\right) & \frac{2}{3}\left(1-e^{-\frac{3}{2} \lambda_{12} t}\right) \\
\frac{1}{3}\left(1-e^{-\frac{3}{2} \lambda_{12} t}\right) & \frac{1}{3}\left(2+e^{-\frac{3}{2} \lambda_{12} t}\right)
\end{array}\right]
$$

\section{Analysis of Reorder Times}

In this section we consider the time of reorders. As the inventory level depletes from $-s+1$ to $-s$, the shopkeeper places an order for $S+s$ items which occurs when type- 1 or of type- 2 customer demand items and the number of backorders is $s-1$. We define $m^{*}$ as the expected time between two successive reorders in equal priority case. Suppose $m$ as the expected time between two successive reorders in priority case (see [15]). Thus

$$
\begin{aligned}
m & =m_{+} \text {(on-hand inventory) }+m_{-} \text {(backorders) } \\
& =\sum_{i \in \Omega_{A}} \frac{1}{\beta_{i}}+\sum_{i=i \in \Omega_{B}} \frac{1}{\beta_{i}} \\
& =\frac{S}{\lambda_{12}}+\frac{s}{\lambda_{1}}
\end{aligned}
$$

The expected time between two successive reorders is then reduced from the priority case while we apply the equal priority policy. The reduced quantity is obtained as $s\left(\frac{1}{\lambda_{1}}-\frac{2}{\lambda_{12}}\right)$. If $m^{L}$ denotes the expected time between two successive reorders in normal case, then it is the lower bound and we obtain

$$
m>m^{*}>m^{L}=\frac{1}{\lambda_{12}}(s+S) .
$$

Suppose $E(R)$ denotes the expected reorder rate and is given by $E(R)=\frac{\lambda_{12}}{2 s+S}$. It agrees the result of [15] when $\mu=0$. If $E\left(R^{\prime}\right)$ is the expected reorder rate under priority scheme, then

$$
\frac{\lambda_{1}}{s+\lambda_{1} \frac{S}{\lambda_{12}}}<\frac{\frac{\lambda_{12}}{2}}{s+\lambda_{1} \frac{S}{2}}
$$

while $\lambda_{2}>\lambda_{1}$ and hence we obtain $E(R)<E\left(R^{\prime}\right)$.

We also note that suppose $0=\zeta_{0}<\zeta_{1}<\zeta_{2}<\cdots$ be the times at which reorders are placed. Since the supply of orders is instantaneous, the on-hand inventory level at time $\zeta_{n}(n=0,1, \ldots)$ is $S$ and $\left\{\zeta_{n}, n=0,1, \ldots\right\}$ is a renewal process. Then the process obtained is a $s+S+1$ states continuous time Markov chain in which the state $-s$ is an instantaneous 
state. The process is obtained by requiring the path functions to be right continuous with states $\{-s+1,-s+2, \ldots, S\}$ and $F(t)$ be the distribution function between reorder times. As such $F(t)$ is same as the distribution function of the time till absorption into the state $-s$, starting from the initial state $S$. Hence, $F(t)$ is the distribution of phase type and $\left\{\zeta_{n}\right\}$ is a renewal process of phase type (PH-renewal processes).

The nonnegative random variable $X$ of reorder times has a phase-type distribution $(\mathrm{PH}-$ distribution) and its distribution function is then given by

$$
F(t)=\operatorname{Pr}\{X \leq t\}=1-\alpha \exp (T t) \mathbf{e}, \quad t \geq 0 .
$$

where $\alpha$ is a substochastic vector of order $S+s$, i.e., $\alpha$ is a row vector, all elements of $\alpha$ are nonnegative, and $\alpha \mathbf{e} \leq 1$, and $T$ is a subgenerator of order $S+s$, i.e., $T$ is a square matrix of order $S+s$ matrix such that $T=$

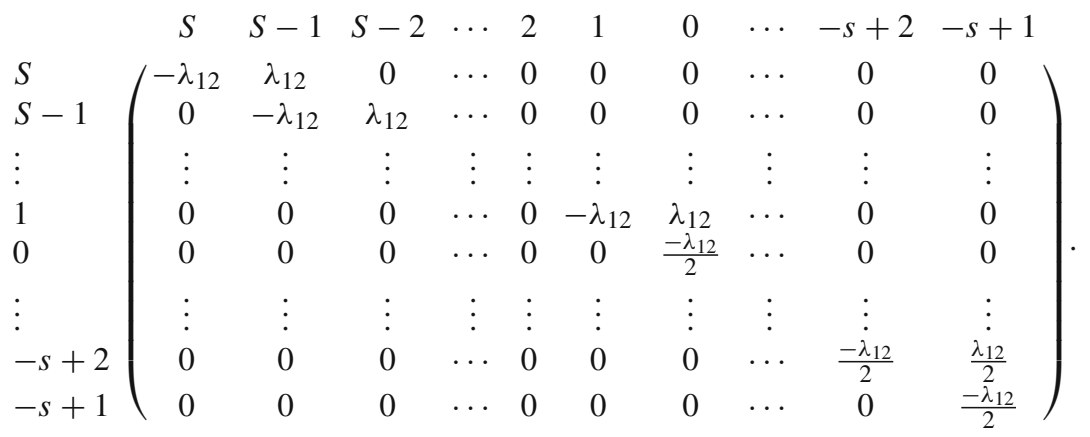

The expected time, $m^{*}$ between two successive reorders is then given by $m^{*}=-\alpha T^{-1} \mathbf{e}$ (see [15]). We assume that

$$
\beta_{i}=\left\{\begin{array}{l}
\lambda_{12} ; i \geq 1 \\
\frac{\lambda_{12} ;}{2} ; i \leq 0
\end{array}\right.
$$

If the process starts at the state $S$, we obtain $\alpha=(1,0,0, \ldots, 0)$, and

$$
m^{*}=\sum_{i} \frac{1}{\beta_{i}}=\frac{S}{\lambda_{12}}+\frac{2 s}{\lambda_{12}}
$$

Suppose the process starts at any one of the states in $\Omega$, the initial probability vector is $\alpha=\frac{1}{S+s} \mathbf{e}_{S+s}^{T}$, then

$$
\begin{aligned}
m^{*} & =\sum_{i}\left(1+\frac{1-i}{n}\right) \frac{1}{\beta_{i}} \\
& =\frac{1}{S+s}\left[S \sum_{i \in \Omega} \frac{1}{\beta_{i}}+\sum_{i \in \Omega} \frac{1-i}{\beta_{i}}\right] \\
& =\frac{1}{2(S+s) \lambda_{12}}[S(S+1)+4(s S)+2 s(s+1)]
\end{aligned}
$$


Once the shopkeeper starts the system with positive inventory level, the value of $\alpha$ is then given by $\left(\frac{1}{S} \mathbf{e}_{S}^{T}, 0,0, \ldots, 0\right)$, and hence

$$
\begin{aligned}
m^{*} & =\frac{1}{S} \sum_{i=1}^{S} \frac{S+1-i}{\beta_{i}}+\sum_{i \in \Omega_{B}} \frac{1}{\beta_{i}} \\
& =\frac{1}{\lambda_{12}}\left[\frac{S+1}{2}+2 s\right] .
\end{aligned}
$$

\section{System Performance Measures}

In this section, we derive several key performance measures of the considered system. We construct the long-run total expected cost per unit time using these measures.

1. Let $E(L)$ denote the expected inventory level at steady state. As $\phi_{i}$ denotes the steady state probability of the number items in the system, the expected inventory level is given by

$$
E(L)=\frac{1}{s+\frac{S}{2}}\left[\frac{S(S+1)}{4}\right] .
$$

2. Let $E(B)$ denote the expected number of backorders in the steady state.

$$
\begin{aligned}
E(B) & =\sum_{i=-s+1}^{-1}-i \phi_{i} \\
& =\frac{s(s-1)}{2} \phi_{0} .
\end{aligned}
$$

3. Suppose $E(R)$ denotes the expected reorder rate, the quantity of it is then given by

$$
E(R)=\frac{\lambda_{12}}{2 s+S} .
$$

4. We observe that the process stays at several states sometimes. The proportion of time that the on-hand inventory level is positive is then given by

$$
\frac{\sum_{i \in \Omega_{A}} \frac{1}{\beta_{i}}}{\sum_{i \in \Omega} \frac{1}{\beta_{i}}}=\frac{\frac{S}{\lambda_{12}}}{\frac{S}{\lambda_{12}}+\frac{s}{\lambda_{12} / 2}}=\frac{S}{S+2 s},
$$

and the proportion of time that the on hand inventory level is negative (backorders) is then given by

$$
\frac{\sum_{i \in \Omega_{B}} \frac{1}{\beta_{i}}}{\sum_{i \in \Omega} \frac{1}{\beta_{i}}}=\frac{2 s}{S+2 s} .
$$

It is noted that the proportion quantities are independent of arrival rates.

5. Let $E(S)$ denote the expected shortage of the customers which is given by

$$
E(S)=\frac{s \lambda_{12}}{2 s+S} .
$$


To study the cost structure of the model, the above system performance measures are used. For this, first we consider the following costs

$h$ : the holding cost per unit item per unit time

$K$ : set-up cost per order

$c_{B}$ : the backorder cost per customer per unit time

$c_{S}$ : the losses in revenue per customer per unit time.

The long-run total expected cost per unit time for this system at steady state is then obtained as follows:

$$
\begin{aligned}
T C(s, S) & =h E(L)+K E(R)+c_{B} E(B)+c_{S} E(S) \\
& =h\left[\frac{1}{\left(s+\frac{S}{2}\right)}\left[\frac{S(S+1)}{4}\right]\right]+K\left[\frac{\lambda_{12}}{2 s+S}\right]+c_{B}\left[\frac{s(s-1)}{2} \phi_{0}\right]+c_{S} \frac{s \lambda_{12}}{2 s+S} \\
& =\frac{1}{\left(s+\frac{S}{2}\right)}\left[\frac{h S(S+1)}{4}+K \frac{\lambda_{12}}{2}+c_{S} \frac{s \lambda_{12}}{2}+c_{B} \frac{s(s-1)}{2}\right] .
\end{aligned}
$$

Now, we must determine the optimal values of $s$ and $S$ to minimise the long-run expected cost per unit time. The following two theorems help us to detect the convex nature of $T C$.

Theorem 1 For a fixed value of backorders, the long-run expected cost rate is pseudo-convex in $S$.

Proof 1 Consider $T_{1}(S)=T C(s, S)$ for any fixed $s$. The cost function is then defined as follows:

$$
T_{1}(S)=\frac{h \frac{S(S+1)}{2}+K_{1}}{s+\frac{S}{2}}=\frac{\alpha(S)}{\beta(S)}(\text { say })
$$

with $K_{1}=\left[K+s c_{S}\right] \frac{\lambda_{12}}{2}+c_{B} \frac{s(s-1)}{2}$.

The first difference $\tilde{T}_{1}(S)$ of $T_{1}(S)$ is $T_{1}(S+1)-T_{1}(S)$ and can be expressed as $\frac{\alpha_{1}(S)}{\beta_{1}(S)}$ where $\alpha_{1}(S)=\alpha(S+1) \beta(S)-\alpha(S) \beta(S+1)$ and $\beta_{1}(S)=\beta(S+1) \beta(S)$. As $\beta_{1}(S)$ is an increasing function of $S$, it is enough to show $\alpha_{1}(S)$ is an increasing function of $S$. The value of $\alpha_{1}(S)$ is a difference of $\left[K_{1}+\frac{h(S+1)(S+2)}{4}\right]\left[s+\frac{S}{2}\right]$ and $\left[K_{1}+\frac{h S(S+1)}{4}\right]\left[s+\frac{S+1}{2}\right]$. Its value is $\frac{h(S+1)}{4}\left[s+\frac{S}{2}\right]-\frac{K_{1}}{2}$. It is an increasing function in $S$. This fact completes the proof.

Theorem 2 For a fixed value of $S$, the long-run expected cost rate is pseudo-convex in $s$.

Proof 2 The proof is simple as in Theorem 1.

\section{Comparison}

In this subsection, we derive the total cost function of the inventory system whereas higher priority is given to the back-order demand class and lower priority is given to the lost-sales demand class in two demand classes models (see [2]). We then compare it with our current model.

As $\frac{\lambda_{12}}{2} \rightarrow \lambda_{2}$ with the value of $\phi_{0}=s+S \frac{\lambda_{1}}{\lambda_{12}}$, the waiting time cost rate is given by

$$
\frac{1}{\left(s+S \frac{\lambda_{1}}{\lambda_{12}}\right)}\left[c_{S} s \lambda_{2}+c_{B} \frac{s(s-1)}{2}\right]
$$


and when $\frac{\lambda_{12}}{2} \rightarrow \lambda_{1}$, the other cost rate is

$$
\frac{1}{\left(s+S \frac{\lambda_{1}}{\lambda_{12}}\right)}\left[\frac{h S(S+1)}{2}\left(\frac{\lambda_{1}}{\lambda_{12}}\right)+K \lambda_{1}\right]
$$

We now define the total cost rate of current model with equal priority by

$$
T C^{E}=\frac{1}{\left(s+\frac{S}{2}\right)}\left[\frac{h S(S+1)}{4}+\left(K+s c_{S}\right) \frac{\lambda_{12}}{2}+c_{B} \frac{s(s-1)}{2}\right]
$$

and by utilizing Eqs. (11) and (12), the total cost rate of existing models is given by

$$
T C=\frac{1}{\left(s+S \frac{\lambda_{1}}{\lambda_{12}}\right)}\left[\frac{h S(S+1)}{2}\left(\frac{\lambda_{1}}{\lambda_{12}}\right)+K \lambda_{1}+c_{S} s \lambda_{2}+c_{B} \frac{s(s-1)}{2}\right] .
$$

Now, we state a lemma to compare the potential values of expected total waiting costs per unit time. It is noted that the intensity of arrival rate of type- 2 is significant on the total cost rate.

Lemma 1 Assumed that $\zeta_{1}=\frac{s}{2}\left[c_{S} s+S c_{S}\right]$ and $\zeta_{2}=\left[\frac{c_{B} s(s-1) S}{4 \lambda_{12}}\right]$. If the arrival rate of type-2 customers is higher than the arrival rate of type-1 customers, then $\zeta_{1}, \zeta_{2}>0$ and the expected waiting cost of $T C^{E}$ is smaller than of $T C$.

Proof 3 We prove this lemma by showing the expected waiting costs of $T C-T C^{E}>0$. Consider the difference of expected waiting costs of these two functions as

$$
\left[s+\frac{S}{2}\right]\left(s c_{S} \lambda_{2}\right)-\left[s+S \frac{\lambda_{1}}{\lambda_{12}}\right]\left(s c_{S} \frac{\lambda_{12}}{2}\right) .
$$

Thus

$$
\frac{s}{2}\left[c_{S} s+S c_{S}\right]\left(\lambda_{2}-\lambda_{1}\right)>0 .
$$

Similarly, the difference of the backorder costs is

$$
c_{B} \frac{s(s-1)}{2}\left[\frac{1}{\left(s+S \frac{\lambda_{1}}{\lambda_{12}}\right)}-\frac{1}{\left(s+\frac{S}{2}\right)}\right]
$$

which is given by

$$
\left[\frac{c_{B} s(s-1) S}{4 \lambda_{12}}\right]\left(\lambda_{2}-\lambda_{1}\right)>0 .
$$

The assumption implies that the Eqs. (15) and (16) are true and this completes the proof.

We now ready to state our main result comparing the waiting costs in the total cost rate.

Theorem 3 Assume that $\zeta_{3}=\frac{s K}{2}$ and $\zeta_{4}=\frac{s h S(S+1) \lambda_{12}}{4}$ and $\rho=\frac{\zeta_{3}+\zeta_{4}}{\zeta_{1}+\zeta_{2}}$. If the arrival rates satisfy the condition of Lemma 1 , the $T C^{E}$ is cost-efficient than the other values of $T C$. 
Proof 4 As in the above Lemma, the comparison of the set-up cost rates yields $\zeta_{3}$ from

$$
\left\{\left[s+\frac{S}{2}\right] K \lambda_{1}-\left[s+S \frac{\lambda_{1}}{\lambda_{12}}\right] K \frac{\lambda_{12}}{2}\right\}\left(\lambda_{1}-\lambda_{2}\right)
$$

and the holding cost rates give $\zeta_{4}$ from the expression

$$
\left\{\left[s+\frac{S}{2}\right] \frac{h S(S+1) \lambda_{1}}{2 \lambda_{12}}-\left[s+S \frac{\lambda_{1}}{\lambda_{12}}\right] \frac{h S(S+1)}{4}\right\}\left(\lambda_{1}-\lambda_{2}\right) .
$$

On collecting the values of $\zeta \mathrm{s}$, as $\rho<1$, the expected total cost rate of $T C^{E}<T C$ when the condition of the Lemma holds, we obtain the result.

\section{Measure on Uncertainty}

The concept of the steady-state of an inventory/queue concerns a probability function, it seems logical to consider a connection between entropy and the uncertainty in this model. Indeed, Shannon entropy [17] considers the probability distribution of signals transmitted over a given communication channel in its argument of uncertainty.

Therefore, it is found feasible and logical that the entropy measure would give the uncertainty of the model behaviour in the long-run. The Shannon entropy for the probability vector $\Phi$ of this mode is defined as

$$
\begin{aligned}
H(\Phi) & =\sum_{i=-s+1}^{S} \phi_{i} \log \frac{1}{\phi_{i}} \\
& =s \phi_{0} \log \frac{1}{\phi_{0}}-\left(S \frac{\phi_{0}}{2}\right) \log \frac{\phi_{0}}{2} \\
& =\phi_{0}\left[\log \left(\frac{1}{\phi_{0}}\right)^{s}+\log \left(\frac{2}{\phi_{0}}\right)^{\frac{s}{2}}\right] \\
& =\phi_{0}\left[\log \left\{\left(2^{\frac{S}{2}}\right)\left(\frac{1}{\phi_{0}}\right)^{s+\frac{S}{2}}\right\}\right]
\end{aligned}
$$

We now define

$$
h\left(\phi_{i}\right)=\frac{1}{\phi_{i}} ; \quad\left(0 \leq \phi_{i} \leq 1\right) .
$$

We note that if $\phi_{i}, i=1,2, \ldots, S$, then $h\left(\phi_{i}\right)$ in (17) will be relatively large and if $\phi_{i}, i=-s+1,-s+2, \ldots, 0$, then $h\left(\phi_{i}\right)$ will be small.

Moreover, the principle of maximum entropy suggests what to do and how should the assignment of probabilities be changed when we happen to know (or learn) something about the non-uniformity of the arrivals. To maximize the entropy among all probability distribution $\Phi$ on the set $\Omega$, we want to optimize (maximize) $H(\Phi)=\sum_{-S+1}^{S} \phi_{i} \log \frac{1}{\phi_{i}}$ with the constraint $\phi_{i} \geq 0$ and $\sum_{i \in \Omega} \phi_{i}=1$. We assume 
Table 1 Summative result of cost and entropy rates

\begin{tabular}{llllll}
\hline Cost rates & $h$ & $K$ & $c_{B}$ & $c_{S}$ & Entropy rate \\
\hline Priority (P) & $\frac{\lambda_{1}}{\lambda_{12}} \frac{S(S+1)}{2}$ & $\lambda_{1}$ & $\frac{s(s-1)}{2}$ & $s \lambda_{2}$ & $\log \left[\left(s+\frac{S \lambda_{1}}{\lambda_{12}}\right)\left(\frac{\lambda_{1}}{\lambda_{12}}\right)^{\frac{S}{2\left(s+S \lambda_{1} / \lambda_{12}\right)}}\right]$ \\
Equal priority (EP) & $\frac{S(S+1)}{4}$ & $\frac{\lambda_{12}}{2}$ & $\frac{s(s-1)}{2}$ & $s \frac{\lambda_{12}}{2}$ & $\log \left[\left(s+\frac{S}{2}\right) 2^{\frac{S}{2(s+S / 2)}}\right]$ \\
Normal (N) & $\frac{S(S+1)}{2}$ & $\lambda_{12}$ & $\frac{s(s-1)}{2}$ & - & $\log (s+S)$ \\
\hline
\end{tabular}

$$
\begin{aligned}
\hat{H}(\Phi) & =H(\Phi, \xi) \\
& =-\left[\sum_{i=-s+1}^{S} \phi_{i} \log \frac{1}{\phi_{i}}\right]+\xi\left(\sum_{i \in \Omega} \phi_{i}-1\right),
\end{aligned}
$$

where $\xi$ is a Lagrange multiplier and the $\phi_{i}$ s are probabilities. Since $\frac{\partial \hat{H}}{\partial \phi_{j}}=-1+\xi+\log \phi_{j}$, so $\phi_{j}=e^{\xi-1}$ for all $j$. It happens on two cases.

Case 1. For $\lambda_{2} \longrightarrow \lambda_{1}, i \in \Omega_{A}$ and $\lambda_{2}=0, i \in \Omega_{B}$, we obtain the maximum entropy distribution $\hat{\Phi}$ which is a discrete uniform distribution of size $s+S$. Since $\lambda_{2}>\lambda_{1}$, the arrival rate of type- 2 customer is reduced.

Case 2. When a quantity of $\frac{\lambda_{12}}{2}$ is added with the arrival rate for $i \in \Omega_{A}$, we also obtain the same maximum entropy distribution $\hat{\Phi}$.

By the results of the cases, we note that

$$
\begin{aligned}
H(\Phi)= & \phi_{0}\left[\log \left\{\left(2^{\frac{S}{2}}\right)\left(\frac{1}{\phi_{0}}\right)^{s+\frac{S}{2}}\right\}\right] \\
& \leq \log (s+S)
\end{aligned}
$$

To compare these results, we tabulate the cost rates and the corresponding entropy rates as follows (Table 1).

The priority case $(\mathbf{P})$ in the table shows the results of cost rates and the corresponding entropy rate. The equal priority (EP) case can be directly obtained from $\mathbf{P}$ as $\lambda_{1} \longrightarrow \frac{\lambda_{12}}{2}$. The entropy rate of $\mathbf{E P}$ is more than the entropy rate of $\mathbf{P}$. Similarly, the normal $(\mathbf{N})$ case can be also obtained from $\mathbf{P}$ as $\lambda_{1} \longrightarrow \lambda_{12}$. The $\mathbf{N}$ has the maximum entropy rate of quantity $\log (s+S)$. Also, we note that the holding and the set-up cost rates are increased while the case $\mathbf{P}$ changes to $\mathbf{E P}$ and then changes to $\mathbf{N}$. On the other hand, the shortage rate is decreased during the stock-out period. This fact can intuitively be explained in terms of lost sales, because the shortage of non-priority customers is reduced.

We now consider the steady state distribution $\tilde{\Phi}$ with non-perishable items which was derived in [15]. The probability distribution $\tilde{\Phi}$ is used to derive the 'relative entropy'. It is a measure of distance between the distributions $\tilde{\Phi}$ and $\Phi$ and is given by 


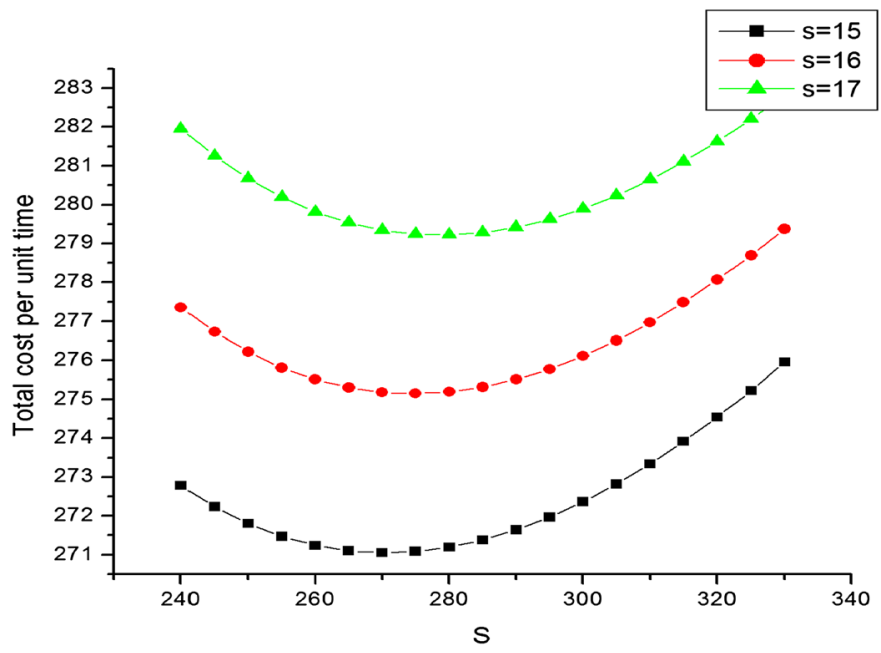

Fig. 1 The optimal value of $S$

$$
\begin{aligned}
\mathbb{D}(\Phi \| \tilde{\Phi})= & \sum_{i \in \Omega} \phi_{i} \log \frac{\phi_{i}}{\phi_{i}^{\prime}} \\
= & s \phi_{0} \log \frac{\phi_{0}}{\phi_{0}^{\prime}}+\frac{\phi_{0}}{2}\left\{\sum_{i \in \Omega_{B}} \log \frac{\left(\frac{\phi_{0}}{2}\right)}{\left(\frac{\phi_{0}^{\prime} \beta_{0}}{\beta_{i}}\right)}\right\} \\
= & s \phi_{0} \log \left\{\frac{s+S\left(\frac{\lambda_{1}}{\lambda_{12}}\right)}{s+\frac{S}{2}}\right\}+\frac{S \phi_{0}}{2} \log \left\{\frac{\left[s+S\left(\frac{\lambda_{1}}{\lambda_{12}}\right)\right] \frac{\lambda_{1}}{\lambda_{12}}}{s+\frac{S}{2}}\right\} \\
= & s \phi_{0} \log \left[s+S \frac{\lambda_{1}}{\lambda_{12}}\right]+\frac{S \phi_{0}}{2} \log \left[\left(s+S \frac{\lambda_{1}}{\lambda_{12}}\right)\left(\frac{\lambda_{1}}{\lambda_{12}}\right)\right] \\
& -\log \left(s+\frac{S}{2}\right)\left[(s+1) \phi_{0}+\frac{S}{2}\right] .
\end{aligned}
$$

\section{Numerical Examples}

In this section, we report our computational experiments on the behaviour of the optimal cost and the optimal solution.

Example 1 In the first example, take the values of parameters and costs as $\lambda_{1}=20 ; \lambda_{2}=$ $50 ; K=300 ; h=1 ; c_{B}=13 ; c_{S}=20$. As in Theorem 1 , we fix the value of $s$ in order to find the optimal value of $S$. By varying the values of $S$, the minimum amount of total cost per unit time $T C^{E}$ is found and its value is 271.05 for $(15,270)$. Figure 1 represents the long run expected total cost rate. It contains three curves together corresponding to $s=15,16$ and 17. We note that the cost is lesser under the higher backorder facility.

The optimal policy of $T C^{E}$ for $s$ is given in Fig. 2. It represents the long run expected total cost rate for $s$ with the same parameter and cost values of the first example. It contains three convex curves together corresponding to $S=20,21$ and 22. As in the first example, we 


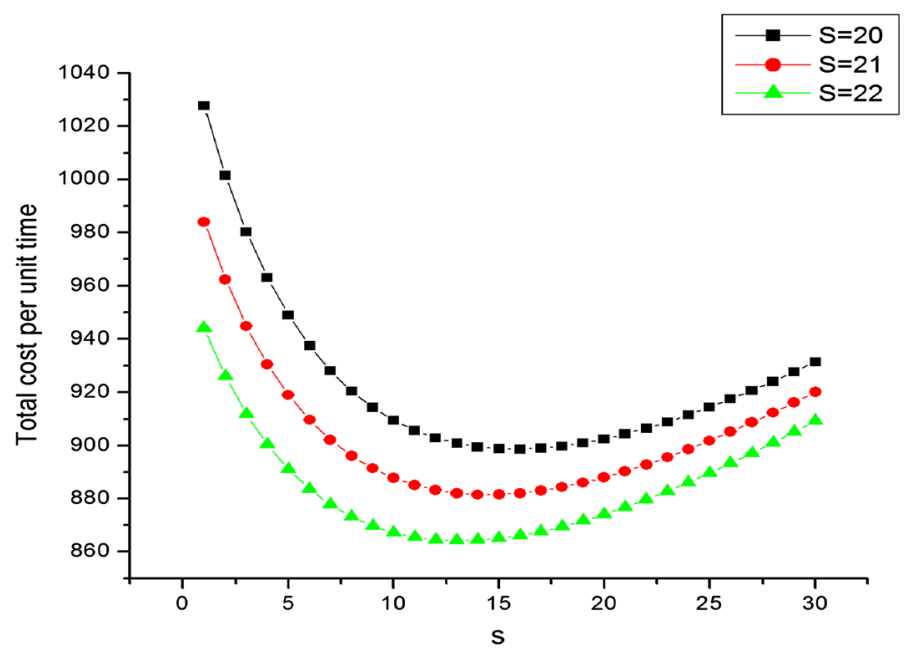

Fig. 2 The optimal value of $s$

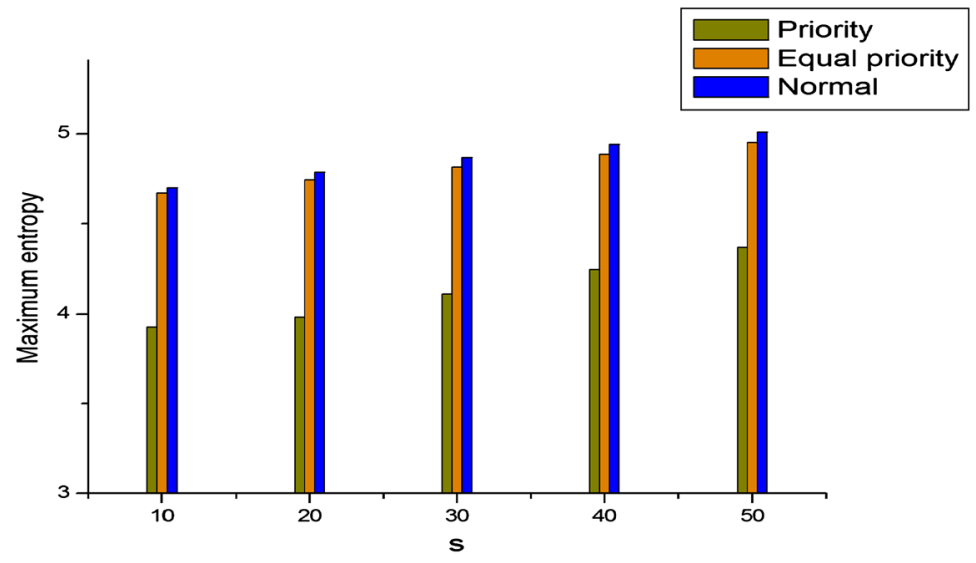

Fig. 3 Maximum entropy for various $s$

fix the value of $S$ and vary the values of $s$. The minimum of total cost per unit time $T C^{E}$ is 864.19 at $(13,22)$. The system with higher stocking facility obtains the minimum cost.

Example 2 We now focus our attention to the maximum entropy obtained in "System performance measures" section. We fix $\lambda_{1}=10$ and $\lambda_{2}=40$ and take the various maximum stocking level and backorders. We have experimented on the entropy rates and compared the maximum entropies for priority, equal priority and normal cases. Figure 3 shows the maximum entropy for the mentioned three cases. The minimum value 3.926059 is obtained at ( $s=10, S=100)$ for priority type and the highest of all the cases is $(=5.010635)$ obtained for normal type. This says the system which attains the higher entropy quantity is more profitable (Fig. 4).

Example 3 In order to evaluate the performance of this change, we compare the expected long run waiting cost rate of $T C^{E}$ with the existing cost structure in the third example. It 


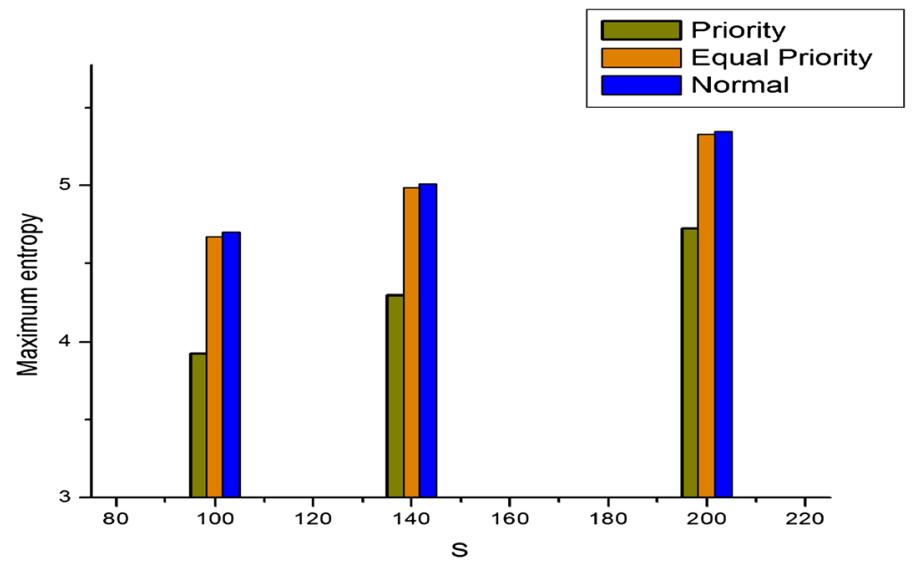

Fig. 4 Influence of $S$ on maximum entropy

Table 2 The relative cost decrease for lower values of $s$

\begin{tabular}{|c|c|c|c|c|c|c|c|}
\hline & \multirow{2}{*}{$\begin{array}{l}s \\
S\end{array}$} & \multicolumn{3}{|l|}{15} & \multicolumn{3}{|l|}{16} \\
\hline & & $T C^{E}$ & $T C$ & $\begin{array}{l}\% \text { of } \\
\text { decrease }\end{array}$ & $T C^{E}$ & $T C$ & $\begin{array}{l}\% \text { of } \\
\text { decrease }\end{array}$ \\
\hline \multirow[t]{11}{*}{$\lambda_{1}=20$ (lower) } & 240 & 87.889 & 195.821 & 55.12 & 93.824 & 207.635 & 54.81 \\
\hline & 241 & 87.565 & 195.153 & 55.13 & 93.480 & 206.936 & 54.83 \\
\hline & 242 & 87.243 & 194.491 & 55.14 & 93.139 & 206.242 & 54.84 \\
\hline & 243 & 86.923 & 193.832 & 55.16 & 92.800 & 205.552 & 54.85 \\
\hline & 244 & 86.606 & 193.179 & 55.17 & 92.464 & 204.867 & 54.87 \\
\hline & 245 & 86.291 & 192.529 & 55.18 & 92.130 & 204.186 & 54.88 \\
\hline & 246 & 85.978 & 191.884 & 55.19 & 91.799 & 203.510 & 54.89 \\
\hline & 247 & 85.668 & 191.244 & 55.20 & 91.470 & 202.838 & 54.91 \\
\hline & 248 & 85.360 & 190.607 & 55.22 & 91.143 & 202.171 & 54.92 \\
\hline & 249 & 85.054 & 189.975 & 55.23 & 90.819 & 201.508 & 54.93 \\
\hline & 250 & 84.750 & 189.347 & 55.24 & 90.496 & 200.850 & 54.94 \\
\hline
\end{tabular}

follows from the proofs 3 and 4 that $T C^{E}<T C$. The main purpose of the experiment is to study the magnitude of this difference as the priority changes, and explore the conditions under which the cost savings from using $T C^{E}$ instead of $T C$ being most significant.

We denote then by $\triangle T C=\frac{T C-T C^{E}}{T C}$, the relative cost decrease when using the modified policy instead of the existing policies. It is noted that when $S$ increases, the values of $T C$ and $T C^{E}$ decrease and increase as $s$ increases. If $\lambda_{1}$ increases, then both the values come together (Tables 2, 3, 4).

The modification seems to perform in all the situations when $\lambda_{1}$ has lower values. The tables show that the difference of arrival parameters work rationing phenomena considerably. In particular whenever $\lambda_{1}$ is extremely low, the relative cost decrease is more satisfiable. It may be noted that these situations are most appropriate for rationing, and that the relative cost decrease will be high. 
Table 3 The relative cost decrease for moderate values of $s$

\begin{tabular}{|c|c|c|c|c|c|c|c|}
\hline & \multirow{2}{*}{$\begin{array}{l}s \\
S\end{array}$} & \multicolumn{3}{|l|}{15} & \multicolumn{3}{|l|}{16} \\
\hline & & $T C^{E}$ & $T C$ & $\begin{array}{l}\% \text { of } \\
\text { decrease }\end{array}$ & $T C^{E}$ & $T C$ & $\begin{array}{l}\% \text { of } \\
\text { decrease }\end{array}$ \\
\hline \multirow{11}{*}{$\begin{array}{l}\lambda_{1}=35 \\
\quad(\text { medium })\end{array}$} & 240 & 104.556 & 143.775 & 27.28 & 111.471 & 152.93 & 27.11 \\
\hline & 241 & 104.170 & 143.257 & 27.28 & 111.062 & 152.384 & 27.12 \\
\hline & 242 & 103.787 & 142.742 & 27.29 & 110.657 & 151.841 & 27.12 \\
\hline & 243 & 103.407 & 142.232 & 27.30 & 110.255 & 151.303 & 27.13 \\
\hline & 244 & 103.029 & 141.724 & 27.30 & 109.855 & 150.768 & 27.14 \\
\hline & 245 & 102.655 & 141.221 & 27.31 & 109.458 & 150.237 & 27.14 \\
\hline & 246 & 102.283 & 140.721 & 27.32 & 109.065 & 149.709 & 27.15 \\
\hline & 247 & 101.913 & 140.224 & 27.32 & 108.674 & 149.185 & 27.16 \\
\hline & 248 & 101.547 & 139.731 & 27.33 & 108.286 & 148.665 & 27.16 \\
\hline & 249 & 101.183 & 139.242 & 27.33 & 107.900 & 148.149 & 27.17 \\
\hline & 250 & 100.821 & 138.756 & 27.34 & 107.518 & 147.636 & 27.17 \\
\hline
\end{tabular}

Table 4 The relative cost decrease for higher values of $s$

\begin{tabular}{|c|c|c|c|c|c|c|c|}
\hline & \multirow{2}{*}{$\begin{array}{l}S \\
S\end{array}$} & \multicolumn{3}{|l|}{15} & \multicolumn{3}{|l|}{16} \\
\hline & & $T C^{E}$ & $T C$ & $\begin{array}{l}\% \text { of } \\
\text { decrease }\end{array}$ & $T C^{E}$ & $T C$ & $\begin{array}{l}\% \text { of } \\
\text { decrease }\end{array}$ \\
\hline \multirow{11}{*}{$\begin{array}{l}\lambda_{1}=35 \\
\quad \text { (higher) }\end{array}$} & 240 & 121.222 & 121.222 & NF & 129.118 & 129.118 & NF \\
\hline & 241 & 120.775 & 120.775 & NF & 128.645 & 128.645 & NF \\
\hline & 242 & 120.331 & 120.331 & NF & 128.175 & 128.175 & NF \\
\hline & 243 & 119.890 & 119.890 & NF & 127.709 & 127.709 & NF \\
\hline & 244 & 119.453 & 119.453 & NF & 127.246 & 127.246 & NF \\
\hline & 245 & 119.018 & 119.018 & NF & 126.787 & 126.787 & $\mathrm{NF}$ \\
\hline & 246 & 118.587 & 118.587 & $\mathrm{NF}$ & 126.331 & 126.331 & $\mathrm{NF}$ \\
\hline & 247 & 118.159 & 118.159 & NF & 125.878 & 125.878 & $\mathrm{NF}$ \\
\hline & 248 & 117.734 & 117.734 & $\mathrm{NF}$ & 125.429 & 125.429 & $\mathrm{NF}$ \\
\hline & 249 & 117.312 & 117.312 & NF & 124.982 & 124.982 & $\mathrm{NF}$ \\
\hline & 250 & 116.893 & 116.893 & $\mathrm{NF}$ & 124.539 & 124.539 & NF \\
\hline
\end{tabular}

NF Not feasible

\section{Conclusion, Observations and Future directions}

In this paper, a two demand classes inventory system was considered with modified service policy. The steady-state and transient solutions were obtained analytically. The expressions for the distribution function of the reorder times were obtained. Several system performance measures were derived and the modified cost structure was analysed. An entropy-based measures were also calculated. We obtain the following managerial suggestions in this analysis:

The modification of the existing models suggests that the total cost per unit time is reduced when the arrival rate of type- 2 (ordinary) customers is greater than the arrival rate of type- 1 
(priority). The reduction of reordering times suggests better service levels to all types of customers. Entropy maximization is equivalent to profit maximization.

It would be interesting to analyze the problem discussed in this article where demands due to one type of customer are not a Markov process. Naturally, with the inclusion of nonPoisson demands, the problem will be more challenging. The model can also be extended to include bulk demand for anyone of two types of demands. We are now working on the above mentioned extensions, and these will be reported in future publications.

Acknowledgments The authors are very thankful to all the three reviewers for their valuable suggestions.

\section{References}

1. Arslan, H., Graves, S.C., Roemer, T.: A single-product inventory model for multiple demand classes. Manag. Sci. 53(9), 1486-1500 (2007)

2. Tang, Y., Xu, D., Zhou, W.: Inventory rationing in a capacitated system with backorders and lost sales. In: Proceedings of the 2007 IEEE-IEEM, 1579-158 (2007)

3. Duran, S., Liu, T., Simchi-Levi, D., Swann, J.L.: Policies utilizing tactical inventory for servicedifferentiated customers. Oper. Res. Lett. 36, 259-264 (2008)

4. Sana, S.S.: The stochastic EOQ model with random sales price. Appl. Math. Comput. 218(2), 239-248 (2011)

5. De, S.K., Sana, S.S.: Backlogging EOQ model for promotional effort and selling price sensitive demandan intuitionistic fuzzy approach. Ann. Oper. Res. 2013, 1-20 (2013)

6. Pal, B., Sana, S.S., Chaudhuri, K.: A distribution-free newsvendor problem with nonlinear holding cost. Int. J. Syst. Sci. 46(7), 1269-1277 (2015)

7. Sarkar, B., Saren, S.: Partial trade-credit policy of retailer with exponentially deteriorating items. Int. J. Appl. Comput. Math. (2014). doi:10.1007/s40819-014-0019-1

8. Khanra, S., Mandal, B., Sarkar, B.: A comparative study between inventory followed by shortages and shortages followed by inventory under trade-credit policy. Int. J. Appl. Comput. Math. (2015). doi:10. 1007/s40819-015-0024-z

9. Cárdenas-Barrón, L.E., Chung, K.J., Treviño-Garza, G.: Celebrating a century of the economic order quantity model in honor of Ford Whitman Harris. Int. J. Prod. Econ. 155, 1-7 (2014)

10. Sana, S.S.: Price sensitive demand with random sales price-a newsboy problem. Int. J. Syst. Sci. 43(3), 491-498 (2012)

11. Sana, S.S., Goyal, S.K.: $(Q, r, L)$ model for stochastic demand with lead-time dependent partial backlogging. Ann. Oper. Res. 2014, 1-12 (2014)

12. Isotupa, K.P.S.: An $(s, Q)$ Markovian inventory system with lost sales and two demand classes. Math. Comput. Model. 43, 687-694 (2006)

13. Zhao, N., Lian, Z.: A queueing-inventory system with two classes of customers. Int. J. Prod. Econ. 129, 225-231 (2011)

14. Liu, M., Feng, M., Wong, C.Y.: Flexible service policies for a Markov inventory system with two demand classes. Int. J. Prod. Econ. 146, 566-574 (2013)

15. Raj Kumar, M.: Transient behaviour and entropy measures on an inventory system with two demand classes. Appl. Math. Comput. 226, 738-753 (2014)

16. Cover, T.M., Thomas, Joy A.: Elements of Information Theory, 2nd edn. Wiley, Hoboken (2006)

17. Shannon, C.E.: A mathematical theory of communication. Bell Syst. Tech. J. 27, 379-423 (1948) 